\title{
SOME NEW GAUSS-JACOBI AND HERMITE-HADAMARD TYPE INEQUALITIES CONCERNING $(n+1)$-DIFFERENTIABLE GENERALIZED $\left(\left(h_{1}, h_{2}\right) ;\left(\eta_{1}, \eta_{2}\right)\right)$-CONVEX MAPPINGS
}

\author{
ARTION KASHURI, ROZANA LIKO AND SILVESTRU SEVER DRAGOMIR
}

\begin{abstract}
In this article, we first introduced a new class of generalized $\left(\left(h_{1}, h_{2}\right) ;\left(\eta_{1}, \eta_{2}\right)\right)$ convex mappings and two interesting lemmas regarding Gauss-Jacobi and Hermite-Hadamard type integral inequalities. By using the notion of generalized $\left(\left(h_{1}, h_{2}\right) ;\left(\eta_{1}, \eta_{2}\right)\right)$-convexity and the first lemma as an auxiliary result, some new estimates with respect to Gauss-Jacobi type integral inequalities are established. Also, using the second lemma, some new estimates with respect to Hermite-Hadamard type integral inequalities via Caputo $k$-fractional derivatives are obtained. It is pointed out that some new special cases can be deduced from main results of the article.
\end{abstract}

\section{Introduction}

The following notations are used throughout this paper. We use $I$ to denote an interval on the real line $\mathbb{R}=(-\infty,+\infty)$. For any subset $K \subseteq \mathbb{R}^{n}$, where $\mathbb{R}^{n}$ is used to denote a $n$-dimensional vector space, $K^{\circ}$ is the interior of $K$. The set of integrable functions on the interval $[a, b]$ is denoted by $L[a, b]$. The set of functions such that $f^{(n)}$ is continuous on the interval $[a, b]$ is denoted by $C^{n}[a, b]$.

The following inequality, named Hermite-Hadamard inequality, is one of the most famous inequalities in the literature for convex functions.

Theorem 1.1. Let $f: I \subseteq \mathbb{R} \longrightarrow \mathbb{R}$ be a convex function on I and $a, b \in I$ with $a<b$. Then the following inequality holds:

$$
f\left(\frac{a+b}{2}\right) \leq \frac{1}{b-a} \int_{a}^{b} f(x) d x \leq \frac{f(a)+f(b)}{2} .
$$

This inequality (1.1) is also known as trapezium inequality.

Received March 7, 2018, accepted April 27, 2018.

2010 Mathematics Subject Classification. Primary: 26A51; Secondary: 26A33, 26D07, 26D10, 26 D15.

Key words and phrases. Hermite-Hadamard inequality, Hölder's inequality, Minkowski inequality, power mean inequality, Caputo $k$-fractional derivatives, $m$-invex.

Corresponding author: Artion Kashuri. 
The trapezium type inequality has remained an area of great interest due to its wide applications in the field of mathematical analysis. For other recent results which generalize, improve and extend the inequality (1.1) through various classes of convex functions interested readers are referred to [[1]-[32], [34], [36], [38], [40]-[44], [48], [51], [52]].

Let us recall some special functions and evoke some basic definitions as follows.

Definition 1.2. The Euler beta function is defined for $a, b>0$ as

$$
\beta(a, b)=\int_{0}^{1} t^{a-1}(1-t)^{b-1} d t=\frac{\Gamma(a) \Gamma(b)}{\Gamma(a+b)} .
$$

Definition 1.3. For $k \in \mathbb{R}^{+}$and $x \in \mathbb{C}$, the $k$-gamma function is defined by

$$
\Gamma_{k}(x)=\lim _{n \rightarrow \infty} \frac{n ! k^{n}(n k)^{\frac{x}{k}-1}}{(x)_{n, k}} .
$$

Its integral representation is given by

$$
\Gamma_{k}(\alpha)=\int_{0}^{\infty} t^{\alpha-1} e^{-\frac{t^{k}}{k}} d t
$$

One can note that

$$
\Gamma_{k}(\alpha+k)=\alpha \Gamma_{k}(\alpha)
$$

For $k=1$, (1.4) gives integral representation of gamma function.

Definition 1.4. For $k \in \mathbb{R}^{+}$and $x, y \in \mathbb{C}$, the $k$-beta function with two parameters $x$ and $y$ is defined as

$$
\beta_{k}(x, y)=\frac{1}{k} \int_{0}^{1} t^{\frac{x}{k}-1}(1-t)^{\frac{y}{k}-1} d t .
$$

For $k=1$, (1.5) gives integral representation of beta function.

Theorem 1.5. Let $x, y>0$, then for $k$-gamma and $k$-beta function the following equality holds:

$$
\beta_{k}(x, y)=\frac{\Gamma_{k}(x) \Gamma_{k}(y)}{\Gamma_{k}(x+y)} .
$$

Definition 1.6. [28] Let $\alpha>0$ and $\alpha \notin\{1,2,3, \ldots\}, n=[\alpha]+1, f \in C^{n}[a, b]$ such that $f^{(n)}$ exists and are continuous on $[a, b]$. The Caputo fractional derivatives of order $\alpha$ are defined as follows:

$$
{ }^{c} D_{a+}^{\alpha} f(x)=\frac{1}{\Gamma(n-\alpha)} \int_{a}^{x} \frac{f^{(n)}(t)}{(x-t)^{\alpha-n+1}} d t, x>a
$$

and

$$
{ }^{c} D_{b-}^{\alpha} f(x)=\frac{(-1)^{n}}{\Gamma(n-\alpha)} \int_{x}^{b} \frac{f^{(n)}(t)}{(t-x)^{\alpha-n+1}} d t, x<b .
$$


If $\alpha=n \in\{1,2,3, \ldots\}$ and usual derivative of order $n$ exists, then Caputo fractional derivative $\left({ }^{c} D_{a+}^{\alpha} f\right)(x)$ coincides with $f^{(n)}(x)$. In particular we have

$$
\left({ }^{c} D_{a+}^{0} f\right)(x)=\left({ }^{c} D_{b-}^{0} f\right)(x)=f(x)
$$

where $n=1$ and $\alpha=0$.

Definition 1.7. [13] Let $\alpha>0, k \geq 1$ and $\alpha \notin\{1,2,3, \ldots\}, n=[\alpha]+1, f \in C^{n}[a, b]$. The Caputo $k$-fractional derivatives of order $\alpha$ are defined as follows:

$$
{ }^{c} D_{a+}^{\alpha, k} f(x)=\frac{1}{k \Gamma_{k}\left(n-\frac{\alpha}{k}\right)} \int_{a}^{x} \frac{f^{(n)}(t)}{(x-t)^{\frac{\alpha}{k}-n+1}} d t, x>a
$$

and

$$
{ }^{c} D_{b-}^{\alpha, k} f(x)=\frac{(-1)^{n}}{k \Gamma_{k}\left(n-\frac{\alpha}{k}\right)} \int_{x}^{b} \frac{f^{(n)}(t)}{(t-x)^{\frac{\alpha}{k}-n+1}} d t, x<b .
$$

Definition 1.8. [50] A set $S \subseteq \mathbb{R}^{n}$ is said to be invex set with respect to the mapping $\eta: S \times S \longrightarrow$ $\mathbb{R}^{n}$, if $x+t \eta(y, x) \in S$ for every $x, y \in S$ and $t \in[0,1]$.

The invex set $S$ is also termed an $\eta$-connected set.

Definition 1.9. [33] Let $h:[0,1] \longrightarrow \mathbb{R}$ be a non-negative function and $h \neq 0$. The function $f$ on the invex set $K$ is said to be $h$-preinvex with respect to $\eta$, if

$$
f(x+t \eta(y, x)) \leq h(1-t) f(x)+h(t) f(y)
$$

for each $x, y \in K$ and $t \in[0,1]$ where $f(\cdot)>0$.

Clearly, when putting $h(t)=t$ in Definition 1.9, $f$ becomes a preinvex function [39]. If the mapping $\eta(y, x)=y-x$ in Definition 1.9, then the non-negative function $f$ reduces to $h$ convex mappings [47].

Definition 1.10. [49] Let $S \subseteq \mathbb{R}^{n}$ be an invex set with respect to $\eta: S \times S \longrightarrow \mathbb{R}^{n}$. A function $f$ : $S \longrightarrow[0,+\infty)$ is said to be $s$-preinvex (or $s$-Breckner-preinvex) with respect to $\eta$ and $s \in(0,1]$, if for every $x, y \in S$ and $t \in[0,1]$,

$$
f(x+t \eta(y, x)) \leq(1-t)^{s} f(x)+t^{s} f(y) .
$$

Definition 1.11. [35] A function $f: K \longrightarrow \mathbb{R}$ is said to be $s$-Godunova-Levin-Dragomir-preinvex functions of second kind, if

$$
f(x+t \eta(y, x)) \leq(1-t)^{-s} f(x)+t^{-s} f(y),
$$

for each $x, y \in K, t \in(0,1)$ and $s \in(0,1]$. 
Definition 1.12. [10] A non-negative function $f: I \subseteq \mathbb{R} \longrightarrow[0,+\infty)$ is said to be $P$-function, if

$$
f(t x+(1-t) y) \leq f(x)+f(y), \quad \forall x, y \in I, t \in[0,1] .
$$

Definition 1.13. [46] Let $f: K \subseteq \mathbb{R} \longrightarrow \mathbb{R}$ be a non-negative function, a function $f: K \longrightarrow \mathbb{R}$ is said to be a $\operatorname{tg} s$-convex function on $K$ if the inequality

$$
f((1-t) x+t y) \leq t(1-t)[f(x)+f(y)]
$$

grips for all $x, y \in K$ and $t \in(0,1)$.

Definition 1.14. [30] A function $f: I \subseteq \mathbb{R} \longrightarrow \mathbb{R}$ is said to $M T$-convex functions, if it is nonnegative and $\forall x, y \in I$ and $t \in(0,1)$ satisfies the subsequent inequality

$$
f(t x+(1-t) y) \leq \frac{\sqrt{t}}{2 \sqrt{1-t}} f(x)+\frac{\sqrt{1-t}}{2 \sqrt{t}} f(y) .
$$

Definition 1.15. [36] A function: $f: I \subseteq \mathbb{R} \longrightarrow \mathbb{R}$ is said to be $m$-MT-convex, if $f$ is positive and for $\forall x, y \in I$, and $t \in(0,1)$, among $m \in(0,1]$, satisfies the following inequality

$$
f(t x+m(1-t) y) \leq \frac{\sqrt{t}}{2 \sqrt{1-t}} f(x)+\frac{m \sqrt{1-t}}{2 \sqrt{t}} f(y) .
$$

For $m=1$, Definition 1.15 reduces to Definition 1.14 .

The concept of $\eta$-convex functions (at the beginning was named by $\varphi$-convex functions), considered in [15], has been introduced as the following.

Definition 1.16. Consider a convex set $I \subseteq \mathbb{R}$ and a bifunction $\eta: f(I) \times f(I) \longrightarrow \mathbb{R}$. A function $f: I \longrightarrow \mathbb{R}$ is called convex with respect to $\eta$ (briefly $\eta$-convex), if

$$
f(\lambda x+(1-\lambda) y) \leq f(y)+\lambda \eta(f(x), f(y)),
$$

is valid for all $x, y \in I$ and $\lambda \in[0,1]$.

Geometrically it says that if a function is $\eta$-convex on $I$, then for any $x, y \in I$, its graph is on or under the path starting from $(y, f(y))$ and ending at $(x, f(y)+\eta(f(x), f(y)))$. If $f(x)$ should be the end point of the path for every $x, y \in I$, then we have $\eta(x, y)=x-y$ and the function reduces to a convex one. For more results about $\eta$-convex functions (see [[8], [9], [14], [15]]).

Definition 1.17. [1] Let $I \subseteq \mathbb{R}$ be an invex set with respect to $\eta_{1}: I \times I \longrightarrow \mathbb{R}$. Consider $f: I \longrightarrow \mathbb{R}$ and $\eta_{2}: f(I) \times f(I) \longrightarrow \mathbb{R}$. The function $f$ is said to be $\left(\eta_{1}, \eta_{2}\right)$-convex if

$$
f\left(x+\lambda \eta_{1}(y, x)\right) \leq f(x)+\lambda \eta_{2}(f(y), f(x)),
$$

is valid for all $x, y \in I$ and $\lambda \in[0,1]$. 
The Gauss-Jacobi type quadrature formula has the following

$$
\int_{a}^{b}(x-a)^{p}(b-x)^{q} f(x) d x=\sum_{k=0}^{+\infty} B_{m, k} f\left(\gamma_{k}\right)+R_{m}^{\star}|f|,
$$

for certain $B_{m, k}, \gamma_{k}$ and rest $R_{m}^{\star}|f|$ (see [45]).

Recently in [29], Liu obtained several integral inequalities for the left-hand side of (1.20) under the Definition 1.12 of $P$-function. Also in [37], Özdemir et al. established several integral inequalities concerning the left-hand side of (1.20) via some kinds of convexity.

Motivated by above works and references therein, the main objective of this article is to establish some new estimates with respect to Gauss-Jacobi and Hermite-Hadamard type integral inequalities using the notion of generalized $\left(\left(h_{1}, h_{2}\right) ;\left(\eta_{1}, \eta_{2}\right)\right)$-convexity and two interesting lemmas as auxiliary results. It is pointed out that some new special cases will be deduced from main results of the article.

\section{Main results}

The following definitions will be used in this section.

Definition 2.1. [11] A set $K \subseteq \mathbb{R}^{n}$ is named as $m$-invex with respect to the mapping $\eta: K \times$ $K \longrightarrow \mathbb{R}^{n}$ for some fixed $m \in(0,1]$, if $m x+t \eta(y, m x) \in K$ grips for each $x, y \in K$ and any $t \in[0,1]$.

Remark 2.2. In Definition 2.1, under certain conditions, the mapping $\eta(y, m x)$ could reduce to $\eta(y, x)$. When $m=1$, we get Definition 1.8 .

We next introduce the concept of generalized $\left(\left(h_{1}, h_{2}\right) ;\left(\eta_{1}, \eta_{2}\right)\right)$-convex mappings.

Definition 2.3. Let $K \subseteq \mathbb{R}$ be an open $m$-invex set with respect to the mapping $\eta_{1}: K \times K \longrightarrow \mathbb{R}$. Suppose $h_{1}, h_{2}:[0,1] \longrightarrow[0,+\infty)$ and $\varphi: I \longrightarrow K$ are continuous. Consider $f: K \longrightarrow(0,+\infty)$ and $\eta_{2}: f(K) \times f(K) \longrightarrow \mathbb{R}$. The mapping $f$ is said to be generalized $\left(\left(h_{1}, h_{2}\right) ;\left(\eta_{1}, \eta_{2}\right)\right)$-convex, if

$$
f\left(m \varphi(x)+t \eta_{1}(\varphi(y), m \varphi(x))\right) \leq\left[m h_{1}(t) f^{r}(x)+h_{2}(t) \eta_{2}\left(f^{r}(y), f^{r}(x)\right)\right]^{\frac{1}{r}}
$$

holds for all $x, y \in I, r \neq 0$, for any $t \in[0,1]$ and some fixed $m \in(0,1]$.

Remark 2.4. In Definition 2.3, if we choose $m=r=1, h_{1}(t)=1, h_{2}(t)=t, \eta_{1}(\varphi(y), m \varphi(x))=$ $\varphi(y)-m \varphi(x), \eta_{2}\left(f^{r}(y), f^{r}(x)\right)=\eta\left(f^{r}(y), f^{r}(x)\right)$ and $\varphi(x)=x, \forall x \in I$, then we get Definition 1.16. Also, in Definition 2.3, if we choose $m=r=1, h_{1}(t)=1, h_{2}(t)=t$ and $\varphi(x)=x, \forall x \in I$, then we get Definition 1.17. Under some suitable choices as we done above, we can get also the Definitions 1.10 and 1.11.

Remark 2.5. For $r=1$, let us discuss some special cases in Definition 2.3 as follows. 
(I) If taking $h_{1}(t)=h_{2}(t)=1$, then we get generalized $\left((m, P) ;\left(\eta_{1}, \eta_{2}\right)\right)$-convex mappings.

(II) If taking $h_{1}(t)=h(1-t), h_{2}(t)=h(t)$, then we get generalized $\left((m, h) ;\left(\eta_{1}, \eta_{2}\right)\right)$-convex mappings.

(III) If taking $h_{1}(t)=(1-t)^{s}, h_{2}(t)=t^{s}$ for $s \in(0,1]$, then we get generalized $\left((m, s) ;\left(\eta_{1}, \eta_{2}\right)\right)$ Breckner-convex mappings.

(IV) If taking $h_{1}(t)=(1-t)^{-s}, h_{2}(t)=t^{-s}$ for $s \in(0,1]$, then we get generalized $\left((m, s) ;\left(\eta_{1}, \eta_{2}\right)\right)$ Godunova-Levin-Dragomir-convex mappings.

(V) If taking $h_{1}(t)=h_{2}(t)=t(1-t)$, then we get generalized $\left((m, t g s) ;\left(\eta_{1}, \eta_{2}\right)\right)$-convex mappings.

(VI) If taking $h_{1}(t)=\frac{\sqrt{1-t}}{2 \sqrt{t}}, h_{2}(t)=\frac{\sqrt{t}}{2 \sqrt{1-t}}$, then we get generalized $\left(m ;\left(\eta_{1}, \eta_{2}\right)\right)-M T$-convex mappings.

It is worth to mention here that to the best of our knowledge all the special cases discussed above are new in the literature.

Let see the following example of a generalized $\left(\left(h_{1}, h_{2}\right) ;\left(\eta_{1}, \eta_{2}\right)\right)$-convex mapping which is not convex.

Example 2.6. Let take $m=r=1, h_{1}(t)=1, h_{2}(t)=t$ and $\varphi$ an identity function. Consider the function $f:[0,+\infty) \longrightarrow[0,+\infty)$ by

$$
f(x)= \begin{cases}x, & 0 \leq x \leq 2 \\ 2, & x>2\end{cases}
$$

Define two bifunctions $\eta_{1}:[0,+\infty) \times[0,+\infty) \longrightarrow \mathbb{R}$ and $\eta_{2}:[0,+\infty) \times[0,+\infty) \longrightarrow[0,+\infty)$ by

$$
\eta_{1}(x, y)= \begin{cases}-y, & 0 \leq y \leq 2 \\ x+y, & y>2\end{cases}
$$

and

$$
\eta_{2}(x, y)= \begin{cases}x+y, & x \leq y \\ 4(x+y), & x>y\end{cases}
$$

Then $f$ is generalized $\left((1, t) ;\left(\eta_{1}, \eta_{2}\right)\right)$-convex mapping. But $f$ is not preinvex with respect to $\eta_{1}$ and also it is not convex (consider $x=0, y=3$ and $t \in(0,1]$ ).

For establishing our first main results regarding some new Gauss-Jacobi type integral inequalities associated with generalized $\left(\left(h_{1}, h_{2}\right) ;\left(\eta_{1}, \eta_{2}\right)\right)$-convexity, we need the following lemma. 
Lemma 2.7. Let $\varphi: I \longrightarrow K$ be a continuous function. Assume that $f: K=[m \varphi(a), m \varphi(a)+$ $\eta(\varphi(b), m \varphi(a))] \longrightarrow \mathbb{R}$ is a continuous mapping on $K^{\circ}$ with respect to $\eta: K \times K \longrightarrow \mathbb{R}$, where $\eta(\varphi(b), m \varphi(a))>0$. Then for some fixed $m \in(0,1]$ and any fixed $p, q>0$, we have

$$
\begin{gathered}
\int_{m \varphi(a)}^{m \varphi(a)+\eta(\varphi(b), m \varphi(a))}(x-m \varphi(a))^{p}(m \varphi(a)+\eta(\varphi(b), m \varphi(a))-x)^{q} f(x) d x \\
=\eta^{p+q+1}(\varphi(b), m \varphi(a)) \int_{0}^{1} t^{p}(1-t)^{q} f(m \varphi(a)+t \eta(\varphi(b), m \varphi(a))) d t .
\end{gathered}
$$

Proof. It is easy to observe that

$$
\begin{gathered}
\int_{m \varphi(a)}^{m \varphi(a)+\eta(\varphi(b), m \varphi(a))}(x-m \varphi(a))^{p}(m \varphi(a)+\eta(\varphi(b), m \varphi(a))-x)^{q} f(x) d x \\
=\eta(\varphi(b), m \varphi(a)) \int_{0}^{1}(m \varphi(a)+t \eta(\varphi(b), m \varphi(a))-m \varphi(a))^{p} \\
\times(m \varphi(a)+\eta(\varphi(b), m \varphi(a))-m \varphi(a)-t \eta(\varphi(b), m \varphi(a)))^{q} f(m \varphi(a)+t \eta(\varphi(b), m \varphi(a))) d t \\
=\eta^{p+q+1}(\varphi(b), m \varphi(a)) \int_{0}^{1} t^{p}(1-t)^{q} f(m \varphi(a)+t \eta(\varphi(b), m \varphi(a))) d t .
\end{gathered}
$$

This completes the proof of the lemma.

Using Lemma 2.7, we now state the following theorems.

Theorem 2.8. Let $k>1$ and $0<r \leq 1$. Suppose $K=\left[m \varphi(a), m \varphi(a)+\eta_{1}(\varphi(b), m \varphi(a))\right] \subseteq \mathbb{R}$ be an open $m$-invex subset with respect to $\eta_{1}: K \times K \longrightarrow \mathbb{R}$ for some fixed $m \in(0,1]$, where $\eta_{1}(\varphi(b), m \varphi(a))>0$. Also, let $h_{1}, h_{2}:[0,1] \longrightarrow[0,+\infty)$ and $\varphi: I \longrightarrow K$ are continuous. Assume that $f: K \longrightarrow(0,+\infty)$ be a mapping on $K^{\circ}$ such that $f \in L(K)$ and $\eta_{2}: f(K) \times f(K) \longrightarrow \mathbb{R}$. If $f^{\frac{k}{k-1}}$ is generalized $\left(\left(h_{1}, h_{2}\right) ;\left(\eta_{1}, \eta_{2}\right)\right)$-convex mapping, then for any fixed $p, q>0$, the following inequality holds:

$$
\begin{aligned}
& \int_{m \varphi(a)}^{m \varphi(a)+\eta_{1}(\varphi(b), m \varphi(a))}(x-m \varphi(a))^{p}\left(m \varphi(a)+\eta_{1}(\varphi(b), m \varphi(a))-x\right)^{q} f(x) d x \\
& \leq \eta_{1}^{p+q+1}(\varphi(b), m \varphi(a)) \sqrt[k]{\beta(k p+1, k q+1)} \\
& \quad \times\left[m f^{\frac{r k}{k-1}}(a) I^{r}\left(h_{1}(t) ; r\right)+\eta_{2}\left(f^{\frac{r k}{k-1}}(b), f^{\frac{r k}{k-1}}(a)\right) I^{r}\left(h_{2}(t) ; r\right)\right]^{\frac{k-1}{r k}},
\end{aligned}
$$

where

$$
I\left(h_{i}(t) ; r\right):=\int_{0}^{1} h_{i}^{\frac{1}{r}}(t) d t, \quad \forall i=1,2 .
$$

Proof. Since $f^{\frac{k}{k-1}}$ is generalized $\left(\left(h_{1}, h_{2}\right) ;\left(\eta_{1}, \eta_{2}\right)\right)$-convex mapping, combining with Lemma 2.7, Hölder inequality, Minkowski inequality and using properties of the modulus, we have

$$
\int_{m \varphi(a)}^{m \varphi(a)+\eta_{1}(\varphi(b), m \varphi(a))}(x-m \varphi(a))^{p}\left(m \varphi(a)+\eta_{1}(\varphi(b), m \varphi(a))-x\right)^{q} f(x) d x
$$




$$
\begin{aligned}
\leq & \left|\eta_{1}(\varphi(b), m \varphi(a))\right|^{p+q+1}\left[\int_{0}^{1} t^{k p}(1-t)^{k q} d t\right]^{\frac{1}{k}} \\
& \times\left[\int_{0}^{1}\left|f\left(m \varphi(a)+t \eta_{1}(\varphi(b), m \varphi(a))\right)\right|^{\frac{k}{k-1}} d t\right]^{\frac{k-1}{k}} \\
\leq & \eta_{1}^{p+q+1}(\varphi(b), m \varphi(a)) \sqrt[k]{\beta(k p+1, k q+1)} \\
& \times\left[\int_{0}^{1}\left[m h_{1}(t) f^{\frac{r k}{k-1}}(a)+h_{2}(t) \eta_{2}\left(f^{\frac{r k}{k-1}}(b), f^{\frac{r k}{k-1}}(a)\right)\right]^{\frac{1}{r}} d t\right]^{\frac{k-1}{k}} \\
\leq & \eta_{1}^{p+q+1}(\varphi(b), m \varphi(a)) \sqrt[k]{\beta(k p+1, k q+1)} \\
& \times\left[\left(\int_{0}^{1} m^{\frac{1}{r}} f^{\frac{k}{k-1}}(a) h_{1}^{\frac{1}{r}}(t) d t\right)^{r}+\left(\int_{0}^{1} \eta_{2}^{\frac{1}{r}}\left(f^{\frac{r k}{k-1}}(b), f^{\frac{r k}{k-1}}(a)\right) h_{2}^{\frac{1}{r}}(t) d t\right)^{r}\right]^{\frac{k-1}{r k}} \\
= & \eta_{1}^{p+q+1}(\varphi(b), m \varphi(a)) \sqrt[k]{\beta(k p+1, k q+1)} \\
& \times\left[m f^{\frac{r k}{k-1}}(a) I^{r}\left(h_{1}(t) ; r\right)+\eta_{2}\left(f^{\frac{r k}{k-1}}(b), f^{\frac{r k}{k-1}}(a)\right) I^{r}\left(h_{2}(t) ; r\right)\right]^{\frac{k-1}{r k}} \cdot
\end{aligned}
$$

So, the proof of this theorem is completed.

We point out some special cases of Theorem 2.8.

Corollary 2.9. In Theorem 2.8 for $k=2$, we have the following inequality:

$$
\begin{aligned}
& \int_{m \varphi(a)}^{m \varphi(a)+\eta_{1}(\varphi(b), m \varphi(a))}(x-m \varphi(a))^{p}\left(m \varphi(a)+\eta_{1}(\varphi(b), m \varphi(a))-x\right)^{q} f(x) d x \\
& \leq \eta_{1}^{p+q+1}(\varphi(b), m \varphi(a)) \sqrt{\beta(2 p+1,2 q+1)} \\
& \quad \times\left[m f^{2 r}(a) I^{r}\left(h_{1}(t) ; r\right)+\eta_{2}\left(f^{2 r}(b), f^{2 r}(a)\right) I^{r}\left(h_{2}(t) ; r\right)\right]^{\frac{1}{2 r}} .
\end{aligned}
$$

Corollary 2.10. In Theorem 2.8 for $h_{1}(t)=h_{2}(t)=1, m=1, \eta_{1}(\varphi(b), m \varphi(a))=\varphi(b)-m \varphi(a)$, $\varphi(x)=x$ and $f(x) \leq L, \forall x \in I$, we get the following inequality:

$$
\int_{a}^{b}(x-a)^{p}(b-x)^{q} f(x) d x \leq(b-a)^{p+q+1} \sqrt[k]{\beta(k p+1, k q+1)} \times\left[L^{\frac{r k}{k-1}}+\eta_{2}\left(L^{\frac{r k}{k-1}}, L^{\frac{r k}{k-1}}\right)\right]^{\frac{k-1}{r k}} .
$$

Corollary 2.11. In Theorem 2.8 for $h_{1}(t)=h(1-t), h_{2}(t)=h(t), m=1, \eta_{1}(\varphi(b), m \varphi(a))=$ $\varphi(b)-m \varphi(a), \varphi(x)=x$ and $f(x) \leq L, \forall x \in I$, we get the following inequality:

$$
\begin{aligned}
& \int_{a}^{b}(x-a)^{p}(b-x)^{q} f(x) d x \\
& \leq(b-a)^{p+q+1} \sqrt[k]{\beta(k p+1, k q+1)} I^{\frac{k-1}{k}}(h(t) ; r)\left[L^{\frac{r k}{k-1}}+\eta_{2}\left(L^{\frac{r k}{k-1}}, L^{\frac{r k}{k-1}}\right)\right]^{\frac{k-1}{r k}} .
\end{aligned}
$$


Corollary 2.12. In Corollary 2.11 for $h_{1}(t)=(1-t)^{s}, h_{2}(t)=t^{s}$, we get the following inequality:

$$
\begin{aligned}
& \int_{a}^{b}(x-a)^{p}(b-x)^{q} f(x) d x \\
& \leq(b-a)^{p+q+1} \sqrt[k]{\beta(k p+1, k q+1)}\left(\frac{r}{r+s}\right)^{\frac{k-1}{k}}\left[L^{\frac{r k}{k-1}}+\eta_{2}\left(L^{\frac{r k}{k-1}}, L^{\frac{r k}{k-1}}\right)\right]^{\frac{k-1}{r k}} .
\end{aligned}
$$

Corollary 2.13. In Corollary 2.11 for $h_{1}(t)=(1-t)^{-s}, h_{2}(t)=t^{-s}$ and $0<s<r$, we get the following inequality:

$$
\begin{aligned}
& \int_{a}^{b}(x-a)^{p}(b-x)^{q} f(x) d x \\
& \leq(b-a)^{p+q+1} \sqrt[k]{\beta(k p+1, k q+1)}\left(\frac{r}{r-s}\right)^{\frac{k-1}{k}}\left[L^{\frac{r k}{k-1}}+\eta_{2}\left(L^{\frac{r k}{k-1}}, L^{\frac{r k}{k-1}}\right)\right]^{\frac{k-1}{r k}} .
\end{aligned}
$$

Corollary 2.14. In Theorem 2.8 for $h_{1}(t)=h_{2}(t)=t(1-t), m=1, \eta_{1}(\varphi(b), m \varphi(a))=\varphi(b)-$ $m \varphi(a), \varphi(x)=x$ and $f(x) \leq L, \forall x \in I$, we get the following inequality:

$$
\begin{aligned}
& \int_{a}^{b}(x-a)^{p}(b-x)^{q} f(x) d x \\
& \leq(b-a)^{p+q+1} \sqrt[k]{\beta(k p+1, k q+1)} \beta^{\frac{k-1}{k}}\left(1+\frac{1}{r}, 1+\frac{1}{r}\right)\left[L^{\frac{r k}{k-1}}+\eta_{2}\left(L^{\frac{r k}{k-1}}, L^{\frac{r k}{k-1}}\right)\right]^{\frac{k-1}{r k}} .
\end{aligned}
$$

Corollary 2.15. In Corollary 2.11 for $h_{1}(t)=\frac{\sqrt{1-t}}{2 \sqrt{t}}, h_{2}(t)=\frac{\sqrt{t}}{2 \sqrt{1-t}}$ and $r \in\left(\frac{1}{2}, 1\right]$, we get the following inequality:

$$
\begin{aligned}
& \int_{a}^{b}(x-a)^{p}(b-x)^{q} f(x) d x \\
& \leq(b-a)^{p+q+1} \sqrt[k]{\beta(k p+1, k q+1)} \beta^{\frac{k-1}{k}}\left(1-\frac{1}{2 r}, 1+\frac{1}{2 r}\right)\left[L^{\frac{r k}{k-1}}+\eta_{2}\left(L^{\frac{r k}{k-1}}, L^{\frac{r k}{k-1}}\right)\right]^{\frac{k-1}{r k}} .
\end{aligned}
$$

Theorem 2.16. Let $l \geq 1$ and $0<r \leq 1$. Suppose $K=\left[m \varphi(a), m \varphi(a)+\eta_{1}(\varphi(b), m \varphi(a))\right] \subseteq \mathbb{R}$ be an open $m$-invex subset with respect to $\eta_{1}: K \times K \longrightarrow \mathbb{R}$ for some fixed $m \in(0,1]$, where $\eta_{1}(\varphi(b), m \varphi(a))>0$. Also, let $h_{1}, h_{2}:[0,1] \longrightarrow[0,+\infty)$ and $\varphi: I \longrightarrow K$ are continuous. Assume that $f: K \longrightarrow(0,+\infty)$ be a mapping on $K^{\circ}$ such that $f \in L_{1}(K)$ and $\eta_{2}: f(K) \times f(K) \longrightarrow \mathbb{R}$. If $f^{l}$ is generalized $\left(\left(h_{1}, h_{2}\right) ;\left(\eta_{1}, \eta_{2}\right)\right)$-convex mapping, then for any fixed $p, q>0$, the following inequality holds:

$$
\begin{aligned}
& \int_{m \varphi(a)}^{m \varphi(a)+\eta_{1}(\varphi(b), m \varphi(a))}(x-m \varphi(a))^{p}\left(m \varphi(a)+\eta_{1}(\varphi(b), m \varphi(a))-x\right)^{q} f(x) d x \\
& \leq \eta_{1}^{p+q+1}(\varphi(b), m \varphi(a)) \beta^{\frac{l-1}{l}}(p+1, q+1) \\
& \quad \times \sqrt[r l]{m f^{r l}(a) I^{r}\left(h_{1}(t) ; p, q, r\right)+\eta_{2}\left(f^{r l}(b), f^{r l}(a)\right) I^{r}\left(h_{2}(t) ; p, q, r\right),}
\end{aligned}
$$

where

$$
I\left(h_{i}(t) ; p, q, r\right):=\int_{0}^{1} t^{p}(1-t)^{q} h_{i}^{\frac{1}{r}}(t) d t, \quad \forall i=1,2 .
$$


Proof. Since $f^{l}$ is generalized $\left(\left(h_{1}, h_{2}\right) ;\left(\eta_{1}, \eta_{2}\right)\right)$-convex mapping, combining with Lemma 2.7, the well-known power mean inequality, Minkowski inequality and using properties of the modulus, we have

$$
\begin{aligned}
\int_{m \varphi(a)}^{m \varphi(a)+\eta_{1}(\varphi(b), m \varphi(a))}(x-m \varphi(a))^{p}\left(m \varphi(a)+\eta_{1}(\varphi(b), m \varphi(a))-x\right)^{q} f(x) d x \\
=\eta_{1}^{p+q+1}(\varphi(b), m \varphi(a)) \int_{0}^{1}\left[t^{p}(1-t)^{q}\right]^{\frac{l-1}{l}}\left[t^{p}(1-t)^{q}\right]^{\frac{1}{l}} f\left(m \varphi(a)+t \eta_{1}(\varphi(b), m \varphi(a))\right) d t \\
\leq\left|\eta_{1}(\varphi(b), m \varphi(a))\right|^{p+q+1}\left[\int_{0}^{1} t^{p}(1-t)^{q} d t\right]^{\frac{l-1}{l}} \\
\quad \times\left[\int_{0}^{1} t^{p}(1-t)^{q}\left|f\left(m \varphi(a)+t \eta_{1}(\varphi(b), m \varphi(a))\right)\right|^{l} d t\right]^{\frac{1}{l}} \\
\leq \eta_{1}^{p+q+1}(\varphi(b), m \varphi(a)) \beta^{\frac{l-1}{l}}(p+1, q+1) \\
\quad \times\left[\int_{0}^{1} t^{p}(1-t)^{q}\left[m h_{1}(t) f^{r l}(a)+h_{2}(t) \eta_{2}\left(f^{r l}(b), f^{r l}(a)\right)\right]^{\frac{1}{r}} d t\right]^{\frac{1}{l}} \\
\leq \eta_{1}^{p+q+1}(\varphi(b), m \varphi(a)) \beta^{\frac{l-1}{l}}(p+1, q+1) \\
\quad \times\left[\left(\int_{0}^{1} m^{\frac{1}{r}} f^{l}(a) t^{p}(1-t)^{q} h_{1}^{\frac{1}{r}}(t) d t\right)^{r}+\left(\int_{0}^{1} \eta_{2}^{\frac{1}{r}}\left(f^{r l}(b), f^{r l}(a)\right) t^{p}(1-t)^{q} h_{2}^{\frac{1}{r}}(t) d t\right)^{r}\right]^{\frac{1}{r l}} \\
=\eta_{1}^{p+q+1}(\varphi(b), m \varphi(a)) \beta^{\frac{l-1}{l}}(p+1, q+1) \\
\quad \times \sqrt[r l]{m f^{r l}(a) I^{r}\left(h_{1}(t) ; p, q, r\right)+\eta_{2}\left(f^{r l}(b), f^{r l}(a)\right) I^{r}\left(h_{2}(t) ; p, q, r\right) .}
\end{aligned}
$$

So, the proof of this theorem is completed.

We point out some special cases of Theorem 2.16.

Corollary 2.17. In Theorem 2.16 for $l=1$, we have the following inequality:

$$
\begin{aligned}
& \int_{m \varphi(a)}^{m \varphi(a)+\eta_{1}(\varphi(b), m \varphi(a))}(x-m \varphi(a))^{p}\left(m \varphi(a)+\eta_{1}(\varphi(b), m \varphi(a))-x\right)^{q} f(x) d x \\
& \quad \leq \eta_{1}^{p+q+1}(\varphi(b), m \varphi(a)) \sqrt[r]{m f^{r}(a) I^{r}\left(h_{1}(t) ; p, q, r\right)+\eta_{2}\left(f^{r}(b), f^{r}(a)\right) I^{r}\left(h_{2}(t) ; p, q, r\right)} .
\end{aligned}
$$

Corollary 2.18. In Theorem 2.16 for $h_{1}(t)=h_{2}(t)=1, m=1, \eta_{1}(\varphi(b), m \varphi(a))=\varphi(b)-m \varphi(a)$, $\varphi(x)=x$ and $f(x) \leq L, \forall x \in I$, we get the following inequality:

$$
\int_{a}^{b}(x-a)^{p}(b-x)^{q} f(x) d x \leq(b-a)^{p+q+1} \beta(p+1, q+1) \sqrt[r l]{L^{r l}+\eta_{2}\left(L^{r l}, L^{r l}\right)} .
$$

Corollary 2.19. In Theorem 2.16 for $h_{1}(t)=h(1-t), h_{2}(t)=h(t), m=1, \eta_{1}(\varphi(b), m \varphi(a))=$ $\varphi(b)-m \varphi(a), \varphi(x)=x$ and $f(x) \leq L, \forall x \in I$, we get the following inequality:

$$
\int_{a}^{b}(x-a)^{p}(b-x)^{q} f(x) d x
$$




$$
\leq(b-a)^{p+q+1} \beta^{\frac{l-1}{l}}(p+1, q+1) \sqrt[r l]{L^{r l} I^{r}(h(t) ; q, p, r)+\eta_{2}\left(L^{r l}, L^{r l}\right) I^{r}(h(t) ; p, q, r)} .
$$

Corollary 2.20. In Corollary 2.19 for $h_{1}(t)=(1-t)^{s}, h_{2}(t)=t^{s}$, we get the following inequality:

$$
\begin{aligned}
& \int_{a}^{b}(x-a)^{p}(b-x)^{q} f(x) d x \\
& \leq(b-a)^{p+q+1} \beta^{\frac{l-1}{l}}(p+1, q+1) \\
& \quad \times \sqrt[r l]{L^{r l} \beta^{r}\left(q+\frac{s}{r}+1, p+1\right)+\eta_{2}\left(L^{r l}, L^{r l}\right) \beta^{r}\left(p+\frac{s}{r}+1, q+1\right)} .
\end{aligned}
$$

Corollary 2.21. In Corollary 2.19 for $h_{1}(t)=(1-t)^{-s}, h_{2}(t)=t^{-s}$ and $0<s \leq r$, we get the following inequality:

$$
\begin{aligned}
\int_{a}^{b}(x-a)^{p}(b-x)^{q} f(x) d x \leq(b-a)^{p+q+1} \beta^{\frac{l-1}{l}}(p+1, q+1) \\
\quad \times \sqrt[r l]{L^{r l} \beta^{r}\left(q-\frac{s}{r}+1, p+1\right)+\eta_{2}\left(L^{r l}, L^{r l}\right) \beta^{r}\left(p-\frac{s}{r}+1, q+1\right)} .
\end{aligned}
$$

Corollary 2.22. In Theorem 2.16 for $h_{1}(t)=h_{2}(t)=t(1-t), m=1, \eta_{1}(\varphi(b), m \varphi(a))=\varphi(b)-$ $m \varphi(a), \varphi(x)=x$ and $f(x) \leq L, \forall x \in I$, we get the following inequality:

$$
\begin{aligned}
& \int_{a}^{b}(x-a)^{p}(b-x)^{q} f(x) d x \\
& \quad \leq(b-a)^{p+q+1} \beta^{\frac{l-1}{l}}(p+1, q+1) \beta^{\frac{1}{l}}\left(p+\frac{1}{r}+1, q+\frac{1}{r}+1\right) \sqrt[r l]{L^{r l}+\eta_{2}\left(L^{r l}, L^{r l}\right)} .
\end{aligned}
$$

Corollary 2.23. In Corollary 2.19 for $h_{1}(t)=\frac{\sqrt{1-t}}{2 \sqrt{t}}, h_{2}(t)=\frac{\sqrt{t}}{2 \sqrt{1-t}}$ and $r \in\left[\frac{1}{2}, 1\right]$, we get the following inequality:

$$
\begin{aligned}
& \int_{a}^{b}(x-a)^{p}(b-x)^{q} f(x) d x \\
& \leq\left(\frac{1}{2}\right)^{\frac{1}{r l}}(b-a)^{p+q+1} \beta^{\frac{l-1}{l}}(p+1, q+1) \\
& \quad \times \sqrt[r l]{L^{r l} \beta^{r}\left(q+\frac{1}{2 r}+1, p-\frac{1}{2 r}+1\right)+\eta_{2}\left(L^{r l}, L^{r l}\right) \beta^{r}\left(p+\frac{1}{2 r}+1, q-\frac{1}{2 r}+1\right)} .
\end{aligned}
$$

For establishing our second main results regarding some new Hermite-Hadamard type integral inequalities associated with generalized $\left(\left(h_{1}, h_{2}\right) ;\left(\eta_{1}, \eta_{2}\right)\right)$-convexity via Caputo $k$ fractional derivatives, we need the following lemma.

Lemma 2.24. Let $\alpha>0, k \geq 1$ and $\alpha \notin\{1,2,3, \ldots\}, n=[\alpha]+1$. Let $\varphi: I \longrightarrow K$ be a continuous function. Suppose $K=[m \varphi(a), m \varphi(a)+\eta(\varphi(b), m \varphi(a))] \subseteq \mathbb{R}$ be an open $m$-invex subset with respect to $\eta: K \times K \longrightarrow \mathbb{R}$ for some fixed $m \in(0,1]$, where $\eta(\varphi(b), m \varphi(a))>0$. Assume that $f:$ 
$K \longrightarrow \mathbb{R}$ is a mapping on $K^{\circ}$ such that $f \in C^{n+1}(K)$. Then we have the following equality for Caputo $k$-fractional derivatives:

$$
\begin{aligned}
& \frac{\eta^{n-\frac{\alpha}{k}}(\varphi(x), m \varphi(a)) f^{(n)}(m \varphi(a)+\eta(\varphi(x), m \varphi(a)))}{\eta(\varphi(b), m \varphi(a))} \\
& \quad-\frac{\eta^{n-\frac{\alpha}{k}}(\varphi(x), m \varphi(b)) f^{(n)}(m \varphi(b)+\eta(\varphi(x), m \varphi(b)))}{\eta(\varphi(b), m \varphi(a))}+(-1)^{n+1} \frac{(n k-\alpha) \Gamma_{k}\left(n-\frac{\alpha}{k}\right)}{\eta(\varphi(b), m \varphi(a))} \\
& \quad \times\left[{ }^{c} D_{(m \varphi(a)+\eta(\varphi(x), m \varphi(a)))^{-}}^{\alpha, k} f(m \varphi(a))-{ }^{c} D_{(m \varphi(b)+\eta(\varphi(x), m \varphi(b)))^{-}}^{\alpha, k} f(m \varphi(b))\right] \\
& =\frac{\eta^{n-\frac{\alpha}{k}+1}(\varphi(x), m \varphi(a))}{\eta(\varphi(b), m \varphi(a))} \int_{0}^{1} t^{n-\frac{\alpha}{k}} f^{(n+1)}(m \varphi(a)+t \eta(\varphi(x), m \varphi(a))) d t \\
& -\frac{\eta^{n-\frac{\alpha}{k}+1}(\varphi(x), m \varphi(b))}{\eta(\varphi(b), m \varphi(a))} \int_{0}^{1} t^{n-\frac{\alpha}{k}} f^{(n+1)}(m \varphi(b)+t \eta(\varphi(x), m \varphi(b))) d t .
\end{aligned}
$$

We denote

$$
\begin{aligned}
& I_{f, \eta, \varphi}(x ; \alpha, k, n, m, a, b) \\
& :=\frac{\eta^{n-\frac{\alpha}{k}+1}(\varphi(x), m \varphi(a))}{\eta(\varphi(b), m \varphi(a))} \int_{0}^{1} t^{n-\frac{\alpha}{k}} f^{(n+1)}(m \varphi(a)+t \eta(\varphi(x), m \varphi(a))) d t \\
& -\frac{\eta^{n-\frac{\alpha}{k}+1}(\varphi(x), m \varphi(b))}{\eta(\varphi(b), m \varphi(a))} \int_{0}^{1} t^{n-\frac{\alpha}{k}} f^{(n+1)}(m \varphi(b)+t \eta(\varphi(x), m \varphi(b))) d t .
\end{aligned}
$$

Proof. Integrating by parts, we get

$$
\begin{aligned}
I_{f, \eta, \varphi}(x ; \alpha, k, n, m, a, b) \\
=\frac{\eta^{n-\frac{\alpha}{k}+1}(\varphi(x), m \varphi(a))}{\eta(\varphi(b), m \varphi(a))}\left[\left.\frac{t^{n-\frac{\alpha}{k}} f^{(n)}(m \varphi(a)+t \eta(\varphi(x), m \varphi(a)))}{\eta(\varphi(x), m \varphi(a))}\right|_{0} ^{1}\right. \\
\left.\quad-\frac{n-\frac{\alpha}{k}}{\eta(\varphi(x), m \varphi(a))} \int_{0}^{1} t^{n-\frac{\alpha}{k}-1} f^{(n)}(m \varphi(a)+t \eta(\varphi(x), m \varphi(a))) d t\right] \\
\quad-\frac{\eta^{n-\frac{\alpha}{k}+1}(\varphi(x), m \varphi(b))}{\eta(\varphi(b), m \varphi(a))}\left[\left.\frac{t^{n-\frac{\alpha}{k}} f^{(n)}(m \varphi(b)+t \eta(\varphi(x), m \varphi(b)))}{\eta(\varphi(x), m \varphi(b))}\right|_{0} ^{1}\right. \\
=-\frac{\eta^{n-\frac{\alpha}{k}}(\varphi(x), m \varphi(a)) f^{(n)}(m \varphi(a)+\eta(\varphi(x), m \varphi(a)))}{\eta(\varphi(b), m \varphi(a))} \\
=-\frac{\eta^{n-\frac{\alpha}{k}}(\varphi(x), m \varphi(b)) f^{(n)}(m \varphi(b)+\eta(\varphi(x), m \varphi(b)))}{\eta(\varphi(b), m \varphi(a))}+(-1)^{n+1} \frac{(n k-\alpha) \Gamma_{k}\left(n-\frac{\alpha}{k}\right)}{\eta(\varphi(b), m \varphi(a))} \\
\quad \times\left[{ }^{c} D_{(m \varphi(a)+\eta(\varphi(x), m \varphi(a)))^{-}}^{\alpha, k} t^{n-\frac{\alpha}{k}-1} f^{(n)}(m \varphi(b)+t \eta(\varphi(x), m \varphi(b))) d t\right] \\
\left.{ }^{c} D_{(m \varphi(b)+\eta(\varphi(x), m \varphi(b)))^{-}}^{\alpha, k} f(m \varphi(b))\right] .
\end{aligned}
$$

This completes the proof of the lemma.

Using Lemma 2.24, we now state the following theorems for the corresponding version for power of $(n+1)$-derivatives. 
Theorem 2.25. Let $\alpha>0, k \geq 1,0<r \leq 1$ and $\alpha \notin\{1,2,3, \ldots\}, n=[\alpha]+1$. Also, let $h_{1}, h_{2}$ : $[0,1] \longrightarrow[0,+\infty)$ and $\varphi: I \longrightarrow K$ are continuous. Suppose $K=\left[m \varphi(a), m \varphi(a)+\eta_{1}(\varphi(b), m \varphi(a))\right]$ $\subseteq \mathbb{R}$ be an open $m$-invex subset with respect to $\eta_{1}: K \times K \longrightarrow \mathbb{R}$ for some fixed $m \in(0,1]$, where $\eta_{1}(\varphi(b), m \varphi(a))>0$. Assume that $f: K \longrightarrow(0,+\infty)$ is a mapping on $K^{\circ}$ such that $f \in C^{n+1}(K)$ and $\eta_{2}: f(K) \times f(K) \longrightarrow \mathbb{R}$. If $\left(f^{(n+1)}(x)\right)^{q}$ is generalized $\left(\left(h_{1}, h_{2}\right) ;\left(\eta_{1}, \eta_{2}\right)\right)$-convex mapping, $q>1, p^{-1}+q^{-1}=1$, then the following inequality for Caputo $k$-fractional derivatives holds:

$$
\begin{aligned}
\left|I_{f, \eta_{1}, \varphi}(x ; \alpha, k, n, m, a, b)\right| \leq \frac{1}{\eta_{1}(\varphi(b), m \varphi(a))} & p \sqrt{\frac{1}{\left(n-\frac{\alpha}{k}\right) p+1}} \\
& \times\left\{\left|\eta_{1}(\varphi(x), m \varphi(a))\right|^{n-\frac{\alpha}{k}+1}\right. \\
& \times \sqrt[r q]{m\left(f^{(n+1)}(a)\right)^{r q} I^{r}\left(h_{1}(t) ; r\right)+\eta_{2}\left(\left(f^{(n+1)}(x)\right)^{r q},\left(f^{(n+1)}(a)\right)^{r q}\right) I^{r}\left(h_{2}(t) ; r\right)} \\
& +\left|\eta_{1}(\varphi(x), m \varphi(b))\right|^{n-\frac{\alpha}{k}+1} \\
& \left.\times \sqrt[r q]{m\left(f^{(n+1)}(b)\right)^{r q} I^{r}\left(h_{1}(t) ; r\right)+\eta_{2}\left(\left(f^{(n+1)}(x)\right)^{r q},\left(f^{(n+1)}(b)\right)^{r q}\right) I^{r}\left(h_{2}(t) ; r\right)}\right\},
\end{aligned}
$$

where $I\left(h_{i}(t) ; r\right)$, for all $i=1,2$, are defined as in Theorem 2.8 .

Proof. From Lemma 2.24, generalized $\left(\left(h_{1}, h_{2}\right) ;\left(\eta_{1}, \eta_{2}\right)\right)$-convexity of $\left(f^{(n+1)}(x)\right)^{q}$, Hölder inequality, Minkowski inequality and properties of the modulus, we have

$$
\begin{aligned}
& \left|I_{f, \eta_{1}, \varphi}(x ; \alpha, k, n, m, a, b)\right| \\
& \leq \frac{\left|\eta_{1}(\varphi(x), m \varphi(a))\right|^{n-\frac{\alpha}{k}+1}}{\left|\eta_{1}(\varphi(b), m \varphi(a))\right|} \int_{0}^{1} t^{n-\frac{\alpha}{k}}\left|f^{(n+1)}\left(m \varphi(a)+t \eta_{1}(\varphi(x), m \varphi(a))\right)\right| d t \\
& +\frac{\left|\eta_{1}(\varphi(x), m \varphi(b))\right|^{n-\frac{\alpha}{k}+1}}{\left|\eta_{1}(\varphi(b), m \varphi(a))\right|} \int_{0}^{1} t^{n-\frac{\alpha}{k}}\left|f^{(n+1)}\left(m \varphi(b)+t \eta_{1}(\varphi(x), m \varphi(b))\right)\right| d t \\
& \leq \frac{\left|\eta_{1}(\varphi(x), m \varphi(a))\right|^{n-\frac{\alpha}{k}+1}}{\eta_{1}(\varphi(b), m \varphi(a))}\left(\int_{0}^{1} t^{\left(n-\frac{\alpha}{k}\right) p} d t\right)^{\frac{1}{p}}\left(\int_{0}^{1}\left(f^{(n+1)}\left(m \varphi(a)+t \eta_{1}(\varphi(x), m \varphi(a))\right)\right)^{q} d t\right)^{\frac{1}{q}} \\
& +\frac{\left|\eta_{1}(\varphi(x), m \varphi(b))\right|^{n-\frac{\alpha}{k}+1}}{\eta_{1}(\varphi(b), m \varphi(a))}\left(\int_{0}^{1} t^{\left(n-\frac{\alpha}{k}\right) p} d t\right)^{\frac{1}{p}}\left(\int_{0}^{1}\left(f^{(n+1)}\left(m \varphi(b)+t \eta_{1}(\varphi(x), m \varphi(b))\right)\right)^{q} d t\right)^{\frac{1}{q}} \\
& \leq \frac{\left|\eta_{1}(\varphi(x), m \varphi(a))\right|^{n-\frac{\alpha}{k}+1}}{\eta_{1}(\varphi(b), m \varphi(a))}\left(\int_{0}^{1} t^{\left(n-\frac{\alpha}{k}\right) p} d t\right)^{\frac{1}{p}} \\
& \times\left(\int_{0}^{1}\left[m h_{1}(t)\left(f^{(n+1)}(a)\right)^{r q}+h_{2}(t) \eta_{2}\left(\left(f^{(n+1)}(x)\right)^{r q},\left(f^{(n+1)}(a)\right)^{r q}\right)\right]^{\frac{1}{r}} d t\right)^{\frac{1}{q}} \\
& +\frac{\left|\eta_{1}(\varphi(x), m \varphi(b))\right|^{n-\frac{\alpha}{k}+1}}{\eta_{1}(\varphi(b), m \varphi(a))}\left(\int_{0}^{1} t^{\left(n-\frac{\alpha}{k}\right) p} d t\right)^{\frac{1}{p}} \\
& \times\left(\int_{0}^{1}\left[m h_{1}(t)\left(f^{(n+1)}(b)\right)^{r q}+h_{2}(t) \eta_{2}\left(\left(f^{(n+1)}(x)\right)^{r q},\left(f^{(n+1)}(b)\right)^{r q}\right)\right]^{\frac{1}{r}} d t\right)^{\frac{1}{q}}
\end{aligned}
$$




$$
\begin{aligned}
& \leq \frac{\left|\eta_{1}(\varphi(x), m \varphi(a))\right|^{n-\frac{\alpha}{k}+1}}{\eta_{1}(\varphi(b), m \varphi(a))}\left(\int_{0}^{1} t^{\left(n-\frac{\alpha}{k}\right) p} d t\right)^{\frac{1}{p}} \\
& \times\left\{\left(\int_{0}^{1} m^{\frac{1}{r}}\left(f^{(n+1)}(a)\right)^{q} h_{1}^{\frac{1}{r}}(t) d t\right)^{r}+\left(\int_{0}^{1} \eta_{2}^{\frac{1}{r}}\left(\left(f^{(n+1)}(x)\right)^{r q},\left(f^{(n+1)}(a)\right)^{r q}\right) h_{2}^{\frac{1}{r}}(t) d t\right)^{r}\right\}^{\frac{1}{r q}} \\
& +\frac{\left|\eta_{1}(\varphi(x), m \varphi(b))\right|^{n-\frac{\alpha}{k}+1}}{\eta_{1}(\varphi(b), m \varphi(a))}\left(\int_{0}^{1} t^{\left(n-\frac{\alpha}{k}\right) p} d t\right)^{\frac{1}{p}} \\
& \times\left\{\left(\int_{0}^{1} m^{\frac{1}{r}}\left(f^{(n+1)}(b)\right)^{q} h_{1}^{\frac{1}{r}}(t) d t\right)^{r}+\left(\int_{0}^{1} \eta_{2}^{\frac{1}{r}}\left(\left(f^{(n+1)}(x)\right)^{r q},\left(f^{(n+1)}(b)\right)^{r q}\right) h_{2}^{\frac{1}{r}}(t) d t\right)^{r}\right\}^{\frac{1}{r q}} \\
& =\frac{1}{\eta_{1}(\varphi(b), m \varphi(a))} \sqrt[p]{\frac{1}{\left(n-\frac{\alpha}{k}\right) p+1}} \\
& \times\left\{\left|\eta_{1}(\varphi(x), m \varphi(a))\right|^{n-\frac{\alpha}{k}+1}\right. \\
& \times \sqrt[r q]{m\left(f^{(n+1)}(a)\right)^{r q} I^{r}\left(h_{1}(t) ; r\right)+\eta_{2}\left(\left(f^{(n+1)}(x)\right)^{r q},\left(f^{(n+1)}(a)\right)^{r q}\right) I^{r}\left(h_{2}(t) ; r\right)} \\
& +\left|\eta_{1}(\varphi(x), m \varphi(b))\right|^{n-\frac{\alpha}{k}+1} \\
& \left.\times \sqrt[r q]{m\left(f^{(n+1)}(b)\right)^{r q} I^{r}\left(h_{1}(t) ; r\right)+\eta_{2}\left(\left(f^{(n+1)}(x)\right)^{r q},\left(f^{(n+1)}(b)\right)^{r q}\right) I^{r}\left(h_{2}(t) ; r\right)}\right\} .
\end{aligned}
$$

So, the proof of this theorem is completed.

We point out some special cases of Theorem 2.25 .

Corollary 2.26. In Theorem 2.25 for $p=q=2$, we have the following Hermite-Hadamard type inequality for generalized $\left(\left(h_{1}, h_{2}\right) ;\left(\eta_{1}, \eta_{2}\right)\right)$-convex mappings via Caputo $k$-fractional derivatives:

$$
\begin{aligned}
& \left|I_{f, \eta_{1}, \varphi}(x ; \alpha, k, n, m, a, b)\right| \leq \frac{1}{\sqrt{2\left(n-\frac{\alpha}{k}\right)+1}} \frac{1}{\eta_{1}(\varphi(b), m \varphi(a))}\left\{\left|\eta_{1}(\varphi(x), m \varphi(a))\right|^{n-\frac{\alpha}{k}+1}\right. \\
& \quad \times \sqrt[2 r]{m\left(f^{(n+1)}(a)\right)^{2 r} I^{r}\left(h_{1}(t) ; r\right)+\eta_{2}\left(\left(f^{(n+1)}(x)\right)^{2 r},\left(f^{(n+1)}(a)\right)^{2 r}\right) I^{r}\left(h_{2}(t) ; r\right)} \\
& \quad+\left|\eta_{1}(\varphi(x), m \varphi(b))\right|^{n-\frac{\alpha}{k}+1} \\
& \left.\quad \times \sqrt[2 r]{m\left(f^{(n+1)}(b)\right)^{2 r} I^{r}\left(h_{1}(t) ; r\right)+\eta_{2}\left(\left(f^{(n+1)}(x)\right)^{2 r},\left(f^{(n+1)}(b)\right)^{2 r}\right) I^{r}\left(h_{2}(t) ; r\right)}\right\} .
\end{aligned}
$$

Corollary 2.27. In Theorem 2.25 for $h_{1}(t)=h_{2}(t)=1$ and $f^{(n+1)}(x) \leq L, \forall x \in I$, we get the following Hermite-Hadamard type inequality for generalized $\left((m, P) ;\left(\eta_{1}, \eta_{2}\right)\right)$-convex mappings via Caputo $k$-fractional derivatives:

$$
\begin{aligned}
& \left|I_{f, \eta_{1}, \varphi}(x ; \alpha, k, n, m, a, b)\right| \leq \frac{1}{\eta_{1}(\varphi(b), m \varphi(a))} \sqrt[p]{\frac{1}{\left(n-\frac{\alpha}{k}\right) p+1}} \\
& \times\left[\left|\eta_{1}(\varphi(x), m \varphi(a))\right|^{n-\frac{\alpha}{k}+1}+\left|\eta_{1}(\varphi(x), m \varphi(b))\right|^{n-\frac{\alpha}{k}+1}\right] \sqrt[r q]{m L^{r q}+\eta_{2}\left(L^{r q}, L^{r q}\right)} .
\end{aligned}
$$


Corollary 2.28. In Theorem 2.25 for $h_{1}(t)=h(1-t), h_{2}(t)=h(t)$ and $f^{(n+1)}(x) \leq L, \forall x \in I$, we get the following Hermite-Hadamard type inequality for generalized $\left((m, h) ;\left(\eta_{1}, \eta_{2}\right)\right)$-convex mappings via Caputo $k$-fractional derivatives:

$$
\begin{aligned}
& \left|I_{f, \eta_{1}, \varphi}(x ; \alpha, k, n, m, a, b)\right| \leq \sqrt[p]{\frac{1}{\left(n-\frac{\alpha}{k}\right) p+1}} \frac{I^{\frac{1}{q}}(h(t) ; r)}{\eta_{1}(\varphi(b), m \varphi(a))} \\
& \quad \times\left[\left|\eta_{1}(\varphi(x), m \varphi(a))\right|^{n-\frac{\alpha}{k}+1}+\left|\eta_{1}(\varphi(x), m \varphi(b))\right|^{n-\frac{\alpha}{k}+1}\right] \sqrt[r q]{m L^{r q}+\eta_{2}\left(L^{r q}, L^{r q}\right)}
\end{aligned}
$$

Corollary 2.29. In Corollary 2.28 for $h_{1}(t)=(1-t)^{s}, h_{2}(t)=t^{s}$, we get the following HermiteHadamard type inequality for generalized $\left((m, s) ;\left(\eta_{1}, \eta_{2}\right)\right)$-Breckner-convex mappings via Caputo $k$-fractional derivatives:

$$
\begin{aligned}
& \left|I_{f, \eta_{1}, \varphi}(x ; \alpha, k, n, m, a, b)\right| \leq \frac{1}{\eta_{1}(\varphi(b), m \varphi(a))}\left(\frac{r}{r+s}\right)^{\frac{1}{q}} \sqrt[p]{\frac{1}{\left(n-\frac{\alpha}{k}\right) p+1}} \\
& \times\left[\left|\eta_{1}(\varphi(x), m \varphi(a))\right|^{n-\frac{\alpha}{k}+1}+\left|\eta_{1}(\varphi(x), m \varphi(b))\right|^{n-\frac{\alpha}{k}+1}\right] \sqrt[r q]{m L^{r q}+\eta_{2}\left(L^{r q}, L^{r q}\right)}
\end{aligned}
$$

Corollary 2.30. In Corollary 2.28 for $h_{1}(t)=(1-t)^{-s}, h_{2}(t)=t^{-s}$ and $0<s<r$, we get the following Hermite-Hadamard type inequality for generalized $\left((m, s) ;\left(\eta_{1}, \eta_{2}\right)\right)$-Godunova-LevinDragomir-convex mappings via Caputo $k$-fractional derivatives:

$$
\begin{aligned}
& \left|I_{f, \eta_{1}, \varphi}(x ; \alpha, k, n, m, a, b)\right| \leq \frac{1}{\eta_{1}(\varphi(b), m \varphi(a))}\left(\frac{r}{r-s}\right)^{\frac{1}{q}} \sqrt[p]{\frac{1}{\left(n-\frac{\alpha}{k}\right) p+1}} \\
& \times\left[\left|\eta_{1}(\varphi(x), m \varphi(a))\right|^{n-\frac{\alpha}{k}+1}+\left|\eta_{1}(\varphi(x), m \varphi(b))\right|^{n-\frac{\alpha}{k}+1}\right] \sqrt[r q]{m L^{r q}+\eta_{2}\left(L^{r q}, L^{r q}\right)} .
\end{aligned}
$$

Corollary 2.31. In Theorem 2.25 for $h_{1}(t)=h_{2}(t)=t(1-t)$ and $f^{(n+1)}(x) \leq L, \forall x \in I$, we get the following Hermite-Hadamard type inequality for generalized $\left((m, \operatorname{tg} s) ;\left(\eta_{1}, \eta_{2}\right)\right)$-convex mappings via Caputo $k$-fractional derivatives:

$$
\begin{aligned}
& \left|I_{f, \eta_{1}, \varphi}(x ; \alpha, k, n, m, a, b)\right| \leq \sqrt[p]{\frac{1}{\left(n-\frac{\alpha}{k}\right) p+1}} \frac{\beta^{\frac{1}{q}}\left(1+\frac{1}{r}, 1+\frac{1}{r}\right)}{\eta_{1}(\varphi(b), m \varphi(a))} \\
& \times\left[\left|\eta_{1}(\varphi(x), m \varphi(a))\right|^{n-\frac{\alpha}{k}+1}+\left|\eta_{1}(\varphi(x), m \varphi(b))\right|^{n-\frac{\alpha}{k}+1}\right] \sqrt[r q]{m L^{r q}+\eta_{2}\left(L^{r q}, L^{r q}\right)} .
\end{aligned}
$$

Corollary 2.32. In Corollary 2.28 for $h_{1}(t)=\frac{\sqrt{1-t}}{2 \sqrt{t}}, h_{2}(t)=\frac{\sqrt{t}}{2 \sqrt{1-t}}$ and $r \in\left(\frac{1}{2}, 1\right]$, we get the following Hermite-Hadamard type inequality for generalized $\left(m ;\left(\eta_{1}, \eta_{2}\right)\right)$-MT-convex mappings via Caputo $k$-fractional derivatives:

$$
\begin{aligned}
& \left|I_{f, \eta_{1}, \varphi}(x ; \alpha, k, n, m, a, b)\right| \leq \sqrt[p]{\frac{1}{\left(n-\frac{\alpha}{k}\right) p+1}} \frac{\beta^{\frac{1}{q}}\left(1-\frac{1}{2 r}, 1+\frac{1}{2 r}\right)}{\eta_{1}(\varphi(b), m \varphi(a))} \\
& \quad \times\left[\left|\eta_{1}(\varphi(x), m \varphi(a))\right|^{n-\frac{\alpha}{k}+1}+\left|\eta_{1}(\varphi(x), m \varphi(b))\right|^{n-\frac{\alpha}{k}+1}\right] \sqrt[r q]{m L^{r q}+\eta_{2}\left(L^{r q}, L^{r q}\right)}
\end{aligned}
$$


Theorem 2.33. Let $\alpha>0, k \geq 1,0<r \leq 1$ and $\alpha \notin\{1,2,3, \ldots\}, n=[\alpha]+1$. Also, let $h_{1}, h_{2}$ : $[0,1] \longrightarrow[0,+\infty)$ and $\varphi: I \longrightarrow K$ are continuous. Suppose $K=\left[m \varphi(a), m \varphi(a)+\eta_{1}(\varphi(b), m \varphi(a))\right] \subseteq$ $\mathbb{R}$ be an open $m$-invex subset with respect to $\eta_{1}: K \times K \longrightarrow \mathbb{R}$ for some fixed $m \in(0,1]$, where $\eta_{1}(\varphi(b), m \varphi(a))>0$. Assume that $f: K \longrightarrow(0,+\infty)$ is a mapping on $K^{\circ}$ such that $f \in C^{n+1}(K)$ and $\eta_{2}: f(K) \times f(K) \longrightarrow \mathbb{R}$. If $\left(f^{(n+1)}(x)\right)^{q}$ is generalized $\left(\left(h_{1}, h_{2}\right) ;\left(\eta_{1}, \eta_{2}\right)\right)$-convex mapping, $q \geq 1$, then the following inequality for Caputo $k$-fractional derivatives holds:

$$
\begin{aligned}
& \left|I_{f, \eta_{1}, \varphi}(x ; \alpha, k, n, m, a, b)\right| \\
& \leq\left(\frac{1}{n-\frac{\alpha}{k}+1}\right)^{1-\frac{1}{q}} \frac{1}{\eta_{1}(\varphi(b), m \varphi(a))}\left\{| \eta _ { 1 } ( \varphi ( x ) , m \varphi ( a ) ) | ^ { n - \frac { \alpha } { k } + 1 } \left[m\left(f^{(n+1)}(a)\right)^{r q} I^{r}\left(h_{1}(t) ; n, \alpha, k, r\right)\right.\right. \\
& \left.\quad+\eta_{2}\left(\left(f^{(n+1)}(x)\right)^{r q},\left(f^{(n+1)}(a)\right)^{r q}\right) I^{r}\left(h_{2}(t) ; n, \alpha, k, r\right)\right]^{\frac{1}{r q}} \\
& \quad+\left|\eta_{1}(\varphi(x), m \varphi(b))\right|^{n-\frac{\alpha}{k}+1}\left[m\left(f^{(n+1)}(b)\right)^{r q} I^{r}\left(h_{1}(t) ; n, \alpha, k, r\right)\right. \\
& \left.\left.\quad+\eta_{2}\left(\left(f^{(n+1)}(x)\right)^{r q},\left(f^{(n+1)}(b)\right)^{r q}\right) I^{r}\left(h_{2}(t) ; n, \alpha, k, r\right)\right]^{\frac{1}{r q}}\right\},
\end{aligned}
$$

where

$$
I\left(h_{i}(t) ; n, \alpha, k, r\right):=\int_{0}^{1} t^{n-\frac{\alpha}{k}} h_{i}^{\frac{1}{r}}(t) d t, \quad \forall i=1,2 .
$$

Proof. From Lemma 2.24, generalized relative semi- $\left(r ; m, h_{1}, h_{2}\right)$-preinvexity of $\left(f^{(n+1)}(x)\right)^{q}$, the well-known power mean inequality, Minkowski inequality and properties of the modulus, we have

$$
\begin{aligned}
& \left|I_{f, \eta_{1}, \varphi}(x ; \alpha, k, n, m, a, b)\right| \\
& \leq \frac{\left|\eta_{1}(\varphi(x), m \varphi(a))\right|^{n-\frac{\alpha}{k}+1}}{\left|\eta_{1}(\varphi(b), m \varphi(a))\right|} \int_{0}^{1} t^{n-\frac{\alpha}{k}}\left|f^{(n+1)}\left(m \varphi(a)+t \eta_{1}(\varphi(x), m \varphi(a))\right)\right| d t \\
& +\frac{\left|\eta_{1}(\varphi(x), m \varphi(b))\right|^{n-\frac{\alpha}{k}+1}}{\left|\eta_{1}(\varphi(b), m \varphi(a))\right|} \int_{0}^{1} t^{n-\frac{\alpha}{k}}\left|f^{(n+1)}\left(m \varphi(b)+t \eta_{1}(\varphi(x), m \varphi(b))\right)\right| d t \\
& \leq \frac{\left|\eta_{1}(\varphi(x), m \varphi(a))\right|^{n-\frac{\alpha}{k}+1}}{\eta_{1}(\varphi(b), m \varphi(a))}\left(\int_{0}^{1} t^{n-\frac{\alpha}{k}} d t\right)^{1-\frac{1}{q}} \\
& \times\left(\int_{0}^{1} t^{n-\frac{\alpha}{k}}\left(f^{(n+1)}\left(m \varphi(a)+t \eta_{1}(\varphi(x), m \varphi(a))\right)\right)^{q} d t\right)^{\frac{1}{q}} \\
& +\frac{\left|\eta_{1}(\varphi(x), m \varphi(b))\right|^{n-\frac{\alpha}{k}+1}}{\eta_{1}(\varphi(b), m \varphi(a))}\left(\int_{0}^{1} t^{n-\frac{\alpha}{k}} d t\right)^{1-\frac{1}{q}} \\
& \times\left(\int_{0}^{1} t^{n-\frac{\alpha}{k}}\left(f^{(n+1)}\left(m \varphi(b)+t \eta_{1}(\varphi(x), m \varphi(b))\right)\right)^{q} d t\right)^{\frac{1}{q}} \\
& \leq \frac{\left|\eta_{1}(\varphi(x), m \varphi(a))\right|^{n-\frac{\alpha}{k}+1}}{\eta_{1}(\varphi(b), m \varphi(a))}\left(\int_{0}^{1} t^{n-\frac{\alpha}{k}} d t\right)^{1-\frac{1}{q}}
\end{aligned}
$$




$$
\begin{aligned}
& \times\left(\int_{0}^{1} t^{n-\frac{\alpha}{k}}\left[m h_{1}(t)\left(f^{(n+1)}(a)\right)^{r q}+h_{2}(t) \eta_{2}\left(\left(f^{(n+1)}(x)\right)^{r q},\left(f^{(n+1)}(a)\right)^{r q}\right)\right]^{\frac{1}{r}} d t\right)^{\frac{1}{q}} \\
& +\frac{\left|\eta_{1}(\varphi(x), m \varphi(b))\right|^{n-\frac{\alpha}{k}+1}}{\eta_{1}(\varphi(b), m \varphi(a))}\left(\int_{0}^{1} t^{n-\frac{\alpha}{k}} d t\right)^{1-\frac{1}{q}} \\
& \times\left(\int_{0}^{1} t^{n-\frac{\alpha}{k}}\left[m h_{1}(t)\left(f^{(n+1)}(b)\right)^{r q}+h_{2}(t) \eta_{2}\left(\left(f^{(n+1)}(x)\right)^{r q},\left(f^{(n+1)}(b)\right)^{r q}\right)\right]^{\frac{1}{r}} d t\right)^{\frac{1}{q}} \\
& \leq \frac{\left|\eta_{1}(\varphi(x), m \varphi(a))\right|^{n-\frac{\alpha}{k}+1}}{\eta_{1}(\varphi(b), m \varphi(a))}\left(\int_{0}^{1} t^{n-\frac{\alpha}{k}} d t\right)^{1-\frac{1}{q}}\left\{\left(\int_{0}^{1} m^{\frac{1}{r}} t^{n-\frac{\alpha}{k}}\left(f^{(n+1)}(a)\right)^{q} h_{1}^{\frac{1}{r}}(t) d t\right)^{r}\right. \\
& \left.+\left(\int_{0}^{1} t^{n-\frac{\alpha}{k}} \eta_{2}^{\frac{1}{r}}\left(\left(f^{(n+1)}(x)\right)^{r q},\left(f^{(n+1)}(a)\right)^{r q}\right) h_{2}^{\frac{1}{r}}(t) d t\right)^{r}\right\}^{\frac{1}{r q}} \\
& +\frac{\left|\eta_{1}(\varphi(x), m \varphi(b))\right|^{n-\frac{\alpha}{k}+1}}{\eta_{1}(\varphi(b), m \varphi(a))}\left(\int_{0}^{1} t^{n-\frac{\alpha}{k}} d t\right)^{1-\frac{1}{q}}\left\{\left(\int_{0}^{1} m^{\frac{1}{r}} t^{n-\frac{\alpha}{k}}\left(f^{(n+1)}(b)\right)^{q} h_{1}^{\frac{1}{r}}(t) d t\right)^{r}\right. \\
& \left.+\left(\int_{0}^{1} t^{n-\frac{\alpha}{k}} \eta_{2}^{\frac{1}{r}}\left(\left(f^{(n+1)}(x)\right)^{r q},\left(f^{(n+1)}(b)\right)^{r q}\right) h_{2}^{\frac{1}{r}}(t) d t\right)^{r}\right\}^{\frac{1}{r q}} \\
& =\left(\frac{1}{n-\frac{\alpha}{k}+1}\right)^{1-\frac{1}{q}} \frac{1}{\eta_{1}(\varphi(b), m \varphi(a))}\left\{| \eta _ { 1 } ( \varphi ( x ) , m \varphi ( a ) ) | ^ { n - \frac { \alpha } { k } + 1 } \left[m\left(f^{(n+1)}(a)\right)^{r q} I^{r}\left(h_{1}(t) ; n, \alpha, k, r\right)\right.\right. \\
& \left.+\eta_{2}\left(\left(f^{(n+1)}(x)\right)^{r q},\left(f^{(n+1)}(a)\right)^{r q}\right) I^{r}\left(h_{2}(t) ; n, \alpha, k, r\right)\right]^{\frac{1}{r q}} \\
& +\left|\eta_{1}(\varphi(x), m \varphi(b))\right|^{n-\frac{\alpha}{k}+1}\left[m\left(f^{(n+1)}(b)\right)^{r q} I^{r}\left(h_{1}(t) ; n, \alpha, k, r\right)\right. \\
& \left.\left.+\eta_{2}\left(\left(f^{(n+1)}(x)\right)^{r q},\left(f^{(n+1)}(b)\right)^{r q}\right) I^{r}\left(h_{2}(t) ; n, \alpha, k, r\right)\right]^{\frac{1}{r q}}\right\} .
\end{aligned}
$$

So, the proof of this theorem is completed.

We point out some special cases of Theorem 2.33.

Corollary 2.34. In Theorem 2.33 for $q=1$, we have the following Hermite-Hadamard type inequality for generalized $\left(\left(h_{1}, h_{2}\right) ;\left(\eta_{1}, \eta_{2}\right)\right)$-convex mappings via Caputo $k$-fractional derivatives:

$$
\begin{aligned}
& \left|I_{f, \eta_{1}, \varphi}(x ; \alpha, k, n, m, a, b)\right| \leq \frac{1}{\eta_{1}(\varphi(b), m \varphi(a))} \\
& \quad \times\left\{| \eta _ { 1 } ( \varphi ( x ) , m \varphi ( a ) ) | ^ { n - \frac { \alpha } { k } + 1 } \left[m\left(f^{(n+1)}(a)\right)^{r} I^{r}\left(h_{1}(t) ; n, \alpha, k, r\right)\right.\right. \\
& \left.\quad+\eta_{2}\left(\left(f^{(n+1)}(x)\right)^{r},\left(f^{(n+1)}(a)\right)^{r}\right) I^{r}\left(h_{2}(t) ; n, \alpha, k, r\right)\right]^{\frac{1}{r}} \\
& \quad+\left|\eta_{1}(\varphi(x), m \varphi(b))\right|^{n-\frac{\alpha}{k}+1}\left[m\left(f^{(n+1)}(b)\right)^{r} I^{r}\left(h_{1}(t) ; n, \alpha, k, r\right)\right.
\end{aligned}
$$




$$
\left.\left.+\eta_{2}\left(\left(f^{(n+1)}(x)\right)^{r},\left(f^{(n+1)}(b)\right)^{r}\right) I^{r}\left(h_{2}(t) ; n, \alpha, k, r\right)\right)^{\frac{1}{r}}\right\} .
$$

Corollary 2.35. In Theorem 2.33 for $h_{1}(t)=h_{2}(t)=1$ and $f^{(n+1)}(x) \leq L, \forall x \in I$, we get the following Hermite-Hadamard type inequality for generalized $\left((m, P) ;\left(\eta_{1}, \eta_{2}\right)\right)$-convex mappings via Caputo $k$-fractional derivatives:

$$
\begin{aligned}
& \left|I_{f, \eta_{1}, \varphi}(x ; \alpha, k, n, m, a, b)\right| \leq \frac{1}{\left(n-\frac{\alpha}{k}+1\right)} \frac{1}{\eta_{1}(\varphi(b), m \varphi(a))} \\
& \quad \times\left[\left|\eta_{1}(\varphi(x), m \varphi(a))\right|^{n-\frac{\alpha}{k}+1}+\left|\eta_{1}(\varphi(x), m \varphi(b))\right|^{n-\frac{\alpha}{k}+1}\right] \sqrt[r q]{m L^{r q}+\eta_{2}\left(L^{r q}, L^{r q}\right)} .
\end{aligned}
$$

Corollary 2.36. In Theorem 2.33 for $h_{1}(t)=h(1-t), h_{2}(t)=h(t)$ and $f^{(n+1)}(x) \leq L, \forall x \in I$, we get the following Hermite-Hadamard type inequality for generalized $\left((m, h) ;\left(\eta_{1}, \eta_{2}\right)\right)$-convex mappings via Caputo $k$-fractional derivatives:

$$
\begin{aligned}
& \left|I_{f, \eta_{1}, \varphi}(x ; \alpha, k, n, m, a, b)\right| \leq\left(\frac{1}{n-\frac{\alpha}{k}+1}\right)^{1-\frac{1}{q}} \frac{1}{\eta_{1}(\varphi(b), m \varphi(a))} \\
& \quad \times\left[\left|\eta_{1}(\varphi(x), m \varphi(a))\right|^{n-\frac{\alpha}{k}+1}+\left|\eta_{1}(\varphi(x), m \varphi(b))\right|^{n-\frac{\alpha}{k}+1}\right] \\
& \quad \times \sqrt[r q]{m L^{r q} I^{r}(h(1-t) ; n, \alpha, k, r)+\eta_{2}\left(L^{r q}, L^{r q}\right) I^{r}(h(t) ; n, \alpha, k, r)} .
\end{aligned}
$$

Corollary 2.37. In Corollary 2.36 for $h_{1}(t)=(1-t)^{s}, h_{2}(t)=t^{s}$, we get the following HermiteHadamard type inequality for generalized $\left((m, s) ;\left(\eta_{1}, \eta_{2}\right)\right)$-Breckner-convex mappings via Caputo $k$-fractional derivatives:

$$
\begin{aligned}
& \left|I_{f, \eta_{1}, \varphi}(x ; \alpha, k, n, m, a, b)\right| \leq\left(\frac{1}{n-\frac{\alpha}{k}+1}\right)^{1-\frac{1}{q}} \frac{1}{\eta_{1}(\varphi(b), m \varphi(a))} \\
& \quad \times\left[\left|\eta_{1}(\varphi(x), m \varphi(a))\right|^{n-\frac{\alpha}{k}+1}+\left|\eta_{1}(\varphi(x), m \varphi(b))\right|^{n-\frac{\alpha}{k}+1}\right] \\
& \quad \times \sqrt[r q]{m L^{r q} \beta^{r}\left(n-\frac{\alpha}{k}+1, \frac{s}{r}+1\right)+\eta_{2}\left(L^{r q}, L^{r q}\right)\left(\frac{1}{n-\frac{\alpha}{k}+\frac{s}{r}+1}\right)^{r}} .
\end{aligned}
$$

Corollary 2.38. In Corollary 2.36 for $h_{1}(t)=(1-t)^{-s}, h_{2}(t)=t^{-s}$ and $0<s<r$, we get the following Hermite-Hadamard type inequality for generalized $\left((m, s) ;\left(\eta_{1}, \eta_{2}\right)\right)$-Godunova-LevinDragomir-convex mappings via Caputo $k$-fractional derivatives:

$$
\begin{aligned}
& \left|I_{f, \eta_{1}, \varphi}(x ; \alpha, k, n, m, a, b)\right| \leq\left(\frac{1}{n-\frac{\alpha}{k}+1}\right)^{1-\frac{1}{q}} \frac{1}{\eta_{1}(\varphi(b), m \varphi(a))} \\
& \quad \times\left[\left|\eta_{1}(\varphi(x), m \varphi(a))\right|^{n-\frac{\alpha}{k}+1}+\left|\eta_{1}(\varphi(x), m \varphi(b))\right|^{n-\frac{\alpha}{k}+1}\right] \\
& \quad \times \sqrt[r q]{m L^{r q} \beta^{r}\left(n-\frac{\alpha}{k}+1,1-\frac{s}{r}\right)+\eta_{2}\left(L^{r q}, L^{r q}\right)\left(\frac{1}{n-\frac{\alpha}{k}-\frac{s}{r}+1}\right)^{r}} .
\end{aligned}
$$


Corollary 2.39. In Theorem 2.33 for $h_{1}(t)=h_{2}(t)=t(1-t)$ and $f^{(n+1)}(x) \leq L, \forall x \in I$, we get the following Hermite-Hadamard type inequality for generalized $\left((m, \operatorname{tg} s) ;\left(\eta_{1}, \eta_{2}\right)\right)$-convex mappings via Caputo $k$-fractional derivatives:

$$
\begin{aligned}
& \left.\left|I_{f, \eta_{1}, \varphi}(x ; \alpha, k, n, m, a, b)\right| \leq\left(\frac{1}{n-\frac{\alpha}{k}+1}\right)\right)^{1-\frac{1}{q}} \frac{\sqrt[q]{\beta\left(n-\frac{\alpha}{k}+\frac{1}{r}+1,1+\frac{1}{r}\right)}}{\eta_{1}(\varphi(b), m \varphi(a))} \\
& \times\left[\left|\eta_{1}(\varphi(x), m \varphi(a))\right|^{n-\frac{\alpha}{k}+1}+\left|\eta_{1}(\varphi(x), m \varphi(b))\right|^{n-\frac{\alpha}{k}+1}\right] \sqrt[r q]{m L^{r q}+\eta_{2}\left(L^{r q}, L^{r q}\right)} .
\end{aligned}
$$

Corollary 2.40. In Corollary 2.36 for $h_{1}(t)=\frac{\sqrt{1-t}}{2 \sqrt{t}}, h_{2}(t)=\frac{\sqrt{t}}{2 \sqrt{1-t}}$ and $r \in\left(\frac{1}{2}, 1\right]$, we get the following Hermite-Hadamard type inequality for generalized $\left(m ;\left(\eta_{1}, \eta_{2}\right)\right)$-MT-convex mappings via Caputo $k$-fractional derivatives:

$$
\begin{aligned}
& \left|I_{f, \eta_{1}, \varphi}(x ; \alpha, k, n, m, a, b)\right| \leq \sqrt[r q]{\frac{1}{2}}\left(\frac{1}{n-\frac{\alpha}{k}+1}\right)^{1-\frac{1}{q}} \frac{1}{\eta_{1}(\varphi(b), m \varphi(a))} \\
& \quad \times\left[\left|\eta_{1}(\varphi(x), m \varphi(a))\right|^{n-\frac{\alpha}{k}+1}+\left|\eta_{1}(\varphi(x), m \varphi(b))\right|^{n-\frac{\alpha}{k}+1}\right] \\
& \quad \times \sqrt[r q]{m L^{r q} \beta^{r}\left(n-\frac{\alpha}{k}-\frac{1}{2 r}+1,1+\frac{1}{2 r}\right)+\eta_{2}\left(L^{r q}, L^{r q}\right) \beta^{r}\left(n-\frac{\alpha}{k}+\frac{1}{2 r}+1,1-\frac{1}{2 r}\right)} .
\end{aligned}
$$

Remark 2.41. For $k=1$, by our Theorems 2.25 and 2.33, we can get some new special HermiteHadamard type inequalities associated with generalized $\left(\left(h_{1}, h_{2}\right) ;\left(\eta_{1}, \eta_{2}\right)\right)$-convex mappings via Caputo fractional derivatives of order $\alpha>0$.

Remark 2.42. Also, applying our Theorems 2.25 and 2.33, for appropriate choices of continuous functions $h_{1}, h_{2}$ and $\eta_{1}, \eta_{2}$, we can deduce some new inequalities using special means associated with generalized $\left(\left(h_{1}, h_{2}\right) ;\left(\eta_{1}, \eta_{2}\right)\right)$-convex mappings.

\section{References}

[1] S. M. Aslani, M. R. Delavar and S. M. Vaezpour, Inequalities of Fejér type related to generalized convex functions with applications, Int. J. Anal. Appl., 16(1), (2018), 38-49.

[2] F. Chen, A note on Hermite-Hadamard inequalities for products of convex functions via Riemann-Liouville fractional integrals, Ital. J. Pure Appl. Math., 33, (2014), 299-306.

[3] M. Adil Khan, Y.-M. Chu, A. Kashuri and R. Liko, Hermite-Hadamard type fractional integral inequalities for $M T_{(r ; g, m, \varphi)}$-preinvex functions, J. Comput. Anal. Appl., 26(8), (2019), 1487-1503,

[4] Y.-M. Chu, M. Adil Khan, T. Ali and S. S. Dragomir, Inequalities for $\alpha$-fractional differentiable functions, J. Inequal. Appl., 2017(93), (2017), pp. 12.

[5] Y.-M. Chu, G. D. Wang and X. H. Zhang, Schur convexity and Hadamard's inequality, Math. Inequal. Appl., 13(4), (2010), 725-731.

[6] Y.-M. Chu, M. Adil Khan, T. U. Khan and T. Ali, Generalizations of Hermite-Hadamard type inequalities for MT -convex functions, J. Nonlinear Sci. Appl., 9(5), (2016), 4305-4316. 
[7] Z. Dahmani, On Minkowski and Hermite-Hadamard integral inequalities via fractional integration, Ann. Funct. Anal., 1(1), (2010), 51-58.

[8] M. R. Delavar and S. S. Dragomir, On $\eta$-convexity, Math. Inequal. Appl., 20, (2017), 203-216.

[9] M. R. Delavar and M. De La Sen, Some generalizations of Hermite-Hadamard type inequalities, SpringerPlus, (2016), 5:1661.

[10] S. S. Dragomir, J. Pečarić and L. E. Persson, Some inequalities of Hadamard type, Soochow J. Math., 21, (1995), 335-341.

[11] T. S. Du, J. G. Liao and Y. J. Li, Properties and integral inequalities of Hadamard-Simpson type for the generalized $(s, m)$-preinvex functions, J. Nonlinear Sci. Appl., 9, (2016), 3112-3126.

[12] G. Farid and A. U. Rehman, Generalizations of some integral inequalities for fractional integrals, Ann. Math. Sil., 31, (2017), pp. 14.

[13] G. Farid, A. Javed and A. U. Rehman, On Hadamard inequalities for $n$-times differentiable functions which are relative convex via Caputo $k$-fractional derivatives, Nonlinear Anal. Forum, To appear.

[14] M. E. Gordji, S. S. Dragomir and M. R. Delavar, An inequality related to $\eta$-convex functions (II), Int. J. Nonlinear Anal. Appl., 6(2), (2016), 26-32.

[15] M. E. Gordji, M. R. Delavar and M. De La Sen, On $\varphi$-convex functions, J. Math. Inequal., Wiss 10(1), (2016), 173-183.

[16] A. Kashuri and R. Liko, On Hermite-Hadamard type inequalities for generalized $(s, m, \varphi)$-preinvex functions via $k$-fractional integrals, Adv. Inequal. Appl., 6, (2017), 1-12.

[17] A. Kashuri and R. Liko, Generalizations of Hermite-Hadamard and Ostrowski type inequalities for $M T_{m}$ preinvex functions, Proyecciones, 36(1), (2017), 45-80.

[18] A. Kashuri and R. Liko, Hermite-Hadamard type fractional integral inequalities for generalized $(r ; s, m, \varphi)$ preinvex functions, Eur. J. Pure Appl. Math., 10(3), (2017), 495-505.

[19] A. Kashuri and R. Liko, Hermite-Hadamard type fractional integral inequalities for twice differentiable generalized $(s, m, \varphi)$-preinvex functions, Konuralp J. Math., 5(2), (2017), 228-238.

[20] A. Kashuri and R. Liko, Hermite-Hadamard type inequalities for generalized $(s, m, \varphi)$-preinvex functions via $k$-fractional integrals, Tbil. Math. J., 10(4), (2017), 73-82.

[21] A. Kashuri and R. Liko, Hermite-Hadamard type fractional integral inequalities for $M T_{(m, \varphi)}$-preinvex functions, Stud. Univ. Babeş-Bolyai, Math., 62(4), (2017), 439-450.

[22] A. Kashuri and R. Liko, Hermite-Hadamard type fractional integral inequalities for twice differentiable generalized beta-preinvex functions, J. Fract. Calc. Appl., 9(1), (2018), 241-252.

[23] M. Adil Khan, T. Ali, S. S. Dragomir and M. Z. Sarikaya, Hermite-Hadamard type inequalities for conformable fractional integrals, Revista de la Real Academia de Ciencias Exactas, FÃnsicas y Naturales. Serie A. Matemáticas, (2017), doi:10.1007/s13398-017-0408-5.

[24] M. Adil Khan, Y.-M. Chu, A. Kashuri, R. Liko and G. Ali, New Hermite-Hadamard inequalities for conformable fractional integrals, J. Funct. Spaces, 2018, Article ID 6928130, pp. 9.

[25] M. Adil Khan, Y.-M. Chu, T. U. Khan and J. Khan, Some new inequalities of Hermite-Hadamard type for s-convex functions with applications, Open Math., 15, (2017), 1414-1430.

[26] M. Adil Khan, Y. Khurshid, T. Ali and N. Rehman, Inequalities for three times differentiable functions, J. Math., Punjab Univ., 48(2), (2016), 35-48.

[27] M. Adil Khan, Y. Khurshid and T. Ali, Hermite-Hadamard inequality for fractional integrals via $\eta$-convex functions, Acta Math. Univ. Comenianae, 79(1), (2017), 153-164.

[28] A. A. Kilbas, H. M. Srivastava and J. J. Trujillo, Theory and applications of fractional differential equations, North-Holland Math. Stud., 204, Elsevier, New York-London, (2006).

[29] W. Liu, New integral inequalities involving beta function via P-convexity, Miskolc Math. Notes, 15(2), (2014), 585-591.

[30] W. Liu, W. Wen and J. Park, Ostrowski type fractional integral inequalities for MT-convex functions, Miskolc Math. Notes, 16(1), (2015), 249-256.

[31] W. Liu, W. Wen and J. Park, Hermite-Hadamard type inequalities for MT-convex functions via classical integrals and fractional integrals, J. Nonlinear Sci. Appl., 9, (2016), 766-777. 
[32] C. Luo, T. S. Du, M. Adil Khan, A. Kashuri and Y. Shen, Some k-fractional integrals inequalities through generalized $\lambda_{\phi m}-M T$-preinvexity, J. Comput. Anal. Appl., 27(4), 690-705, 2019.

[33] M. Matłoka, Inequalities for h-preinvex functions, Appl. Math. Comput., 234, (2014), 52-57.

[34] S. Mubeen and G. M. Habibullah, k-Fractional integrals and applications, Int. J. Contemp. Math. Sci., 7, (2012), 89-94.

[35] M. A. Noor, K. I. Noor, M. U. Awan and S. Khan, Hermite-Hadamard inequalities for s-Godunova-Levin preinvex functions, J. Adv. Math. Stud., 7(2), (2014), 12-19.

[36] O. Omotoyinbo and A. Mogbodemu, Some new Hermite-Hadamard integral inequalities for convex functions, Int. J. Sci. Innovation Tech., 1(1), (2014), 1-12.

[37] M. E. Özdemir, E. Set and M. Alomari, Integral inequalities via several kinds of convexity, Creat. Math. Inform. 20(1), (2011), 62-73.

[38] C. Peng, C. Zhou and T. S. Du, Riemann-Liouville fractional Simpson's inequalities through generalized $\left(m, h_{1}, h_{2}\right)$-preinvexity, Ital. J. Pure Appl. Math., 38, (2017), 345-367.

[39] R. Pini, Invexity and generalized convexity, Optimization, 22, (1991), 513-525.

[40] E. Set and A. Gözpinar, A study on Hermite-Hadamard type inequalities for s-convex functions via conformable fractional integrals, Submitted.

[41] E. Set, A. Gözpinar and J. Choi, Hermite-Hadamard type inequalities for twice differentiable m-convex functions via conformable fractional integrals, Far East J. Math. Sci., 101(4), (2017), 873-891.

[42] E. Set, S. S. Karataş, and M. Adil Khan, Hermite-Hadamard type inequalities obtained via fractional integral for differentiable $m$-convex and $(\alpha, m)$-convex functions, Int. J. Anal., 2016, Article ID 4765691, 8 pages.

[43] E. Set, M. Z. Sarikaya and A. Gözpinar, Some Hermite-Hadamard type inequalities for convex functions via conformable fractional integrals and related inequalities, Creat. Math. Inform., 26(2), (2017), 221-229.

[44] H. N. Shi, Two Schur-convex functions related to Hadamard-type integral inequalities, Publ. Math. Debrecen, 78(2), (2011), 393-403.

[45] D. D. Stancu, G. Coman and P. Blaga, Analiză numerică şi teoria aproximării, Cluj-Napoca: Presa Universitară Clujeană., 2, (2002).

[46] M. Tunç, E. Göv and Ü. Şanal, On tgs-convex function and their inequalities, Facta Univ. Ser. Math. Inform., 30(5), (2015), 679-691.

[47] S. Varošanec, On h-convexity, J. Math. Anal. Appl., 326(1), (2007), 303-311.

[48] H. Wang, T. S. Du and Y. Zhang, $k$-fractional integral trapezium-like inequalities through $(h, m)$-convex and ( $\alpha, m)$-convex mappings, J. Inequal. Appl., 2017(311), (2017), pp. 20.

[49] Y. Wang, S. H. Wang and F. Qi, Simpson type integral inequalities in which the power of the absolute value of the first derivative of the integrand is s-preinvex, Facta Univ. Ser. Math. Inform., 28(2), (2013), 151-159.

[50] T. Weir and B. Mond, Preinvex functions in multiple objective optimization, J. Math. Anal. Appl., 136, (1988), 29-38.

[51] X. M. Zhang, Y. M. Chu and X. H. Zhang, The Hermite-Hadamard type inequality of GA-convex functions and its applications, J. Inequal. Appl., (2010), Article ID 507560, pp. 11.

[52] Y. Zhang, T. S. Du, H. Wang, Y. J. Shen and A. Kashuri, Extensions of different type parameterized inequalities for generalized $(m, h)$-preinvex mappings via $k$-fractional integrals, J. Inequal. Appl., 2018(49), (2018), pp. 30.

Department of Mathematics, Faculty of Technical Science, University Ismail Qemali, Vlora, Albania.

E-mail: artionkashuri@gmail.com

Department of Mathematics, Faculty of Technical Science, University Ismail Qemali, Vlora, Albania.

E-mail: rozanaliko86@gmail.com

School of Engineering \& Science, Victoria University, PO Box 14428, Melbourne City, MC 8001, Australia.

E-mail: sever.dragomir@vu.edu.au 